\title{
SPDEF regulates goblet cell hyperplasia in the airway epithelium
}

\author{
Kwon-Sik Park, ${ }^{1}$ Thomas R. Korfhagen, ${ }^{1}$ Michael D. Bruno, ${ }^{1}$ Joseph A. Kitzmiller, ${ }^{1}$ Huajing Wan, ${ }^{1}$ \\ Susan E. Wert, ${ }^{1}$ Gurjit K. Khurana Hershey, ${ }^{2}$ Gang Chen, ${ }^{1}$ and Jeffrey A. Whitsett ${ }^{1}$ \\ 1Division of Pulmonary Biology and 2Division of Allergy and Immunology, Cincinnati Children's Hospital Medical Center \\ and University of Cincinnati College of Medicine, Cincinnati, Ohio, USA.
}

\begin{abstract}
Goblet cell hyperplasia and mucous hypersecretion contribute to the pathogenesis of chronic pulmonary diseases including cystic fibrosis, asthma, and chronic obstructive pulmonary disease. In the present work, mouse SAM pointed domain-containing ETS transcription factor (SPDEF) mRNA and protein were detected in subsets of epithelial cells lining the trachea, bronchi, and tracheal glands. SPDEF interacted with the C-terminal domain of thyroid transcription factor 1, activating transcription of genes expressed selectively in airway epithelial cells, including Sftpa, Scgb1a1, Foxj1, and Sox17. Expression of Spdef in the respiratory epithelium of adult transgenic mice caused goblet cell hyperplasia, inducing both acidic and neutral mucins in vivo, and stainined for both acidic and neutral mucins in vivo. SPDEF expression was increased at sites of goblet cell hyperplasia caused by IL-13 and dust mite allergen in a process that was dependent upon STAT-6. SPDEF was induced following intratracheal allergen exposure and after Th2 cytokine stimulation and was sufficient to cause goblet cell differentiation of Clara cells in vivo.
\end{abstract}

\section{Introduction}

The respiratory tract is lined by diverse populations of epithelial cells that vary during development and along both cephalocaudal and ventral-dorsal axes of the lung. The numbers and characteristics of respiratory epithelial cells change dynamically during lung morphogenesis and in response to injury, infection, and allergens. Conducting airways and trachea-bronchial glands are lined by a number of distinct cell types that include basal, nonciliated secretory (e.g., Clara cells and goblet cells), ciliated columnar, neuroepithelial, mucous, and serous cells. During chronic injury, epithelial cells lining the lung undergo metaplasia/hyperplasia associated with goblet cell hyperplasia and mucous hypersecretion, which are mediated by various cytokines and growth factors including IL-4, IL-13, and inducers of EGF signaling (1-3). Mucous cell hyperplasia is often associated with chronic respiratory disorders, including asthma, chronic obstructive pulmonary disease, and cystic fibrosis. To our knowledge, transcriptional mechanisms controlling goblet cell differentiation have not been previously identified.

Gene targeting and expression studies demonstrate important roles for transcription factors thyroid transcription factor 1 (TTF-1), GATA binding protein 6 (GATA-6), forkhead box J1 (FOXJ1), FOXA2, nuclear factor- 1 (NF-1), and $\beta$-catenin in the regulation of gene expression and differentiation of cells lining the respiratory tract (4-12). TTF-1 plays a critical role in lung morphogenesis - as it is required for formation of the lung parenchyma - and is also necessary for expression of a number of target genes expressed selectively in the lung, including surfactant protein ( $S f t p)$ genes $S f t p a$,

Nonstandard abbreviations used: CCSP, Clara cell secretory protein; ERM, ETS variant gene 5; FOX, forkhead box; GST, glutathione-S-transferase; $M U C 5 \mathrm{~A} / \mathrm{C}$, mucin 5, subtypes $A$ and $C$; PSA, prostate-specific antigen; rtTA, reverse tetracycline transactivator; Scgb, secretoglobin; Sftp, surfactant protein; SOX17, SRY-box containing gene 17; SPDEF, SAM pointed domain-containing ETS transcription factor; TRE2, tetracycline response element 2; TTF-1, thyroid transcription factor 1 .

Conflict of interest: The authors have declared that no conflict of interest exists. Citation for this article: J. Clin. Invest. 117:978-988 (2007). doi:10.1172/JCI29176.
Sftpb, secretoglobin (Scgb) gene, and Scgbla1 (6, 13-16). The activity of TTF-1 is highly dependent upon its interactions with other transcription factors and coactivators, including GATA-6, NF-1, RAR, CBP/p300, SRC-1, and TAZ (4, 17-21). Normal differentiation of the peripheral respiratory epithelium in late gestation and its maintenance in the postnatal period also require the activity of TTF-1 and the Fox family of transcription factors, including FOXA1, FOXA2, and FOXJ1 $(5,9,11,22,23)$. FOXA2 is required for maintenance of normal differentiation of conducting airway epithelial cells, as FOXA2 deletion causes goblet cell hyperplasia in vivo. Loss of FOXA2 is associated with exposure to allergens and cytokines, which induce goblet cell hyperplasia in the mouse (9). While the requirements for TTF-1, FOXA2, and $\beta$-catenin for differentiation and regulation of peripheral airway epithelial cell gene expression are well established, the transcriptional programs controlling differentiation of proximal airway epithelial cells, including those mediating goblet cell hyperplasia, are not well understood.

SAM pointed domain-containing ETS transcription factor (SPDEF) was first described as a member of the ETS family of transcription factors expressed in relatively high abundance in prostate $(24,25)$ and was initially referred to as prostate-specific ETS (Pse) or prostate-derived ETS factor (PDEF). In initial studies, Spdef mRNA was detected in relative abundance in prostate epithelium, intestine, and oviduct in the mouse (25) and in prostate and ovary in humans (24). Human SPDEF interacts with the androgen receptor to enhance expression of the prostate-specific antigen (PSA) promoter in vitro (24). Mouse SPDEF activated the promoter of the MASPIN gene that is expressed in normal epithelial cells in the prostate (25). While SPDEF is expressed in epithelial cells in various tissues, its role in cell differentiation or function remains unknown. In analysis of genes influenced by a phosphorylation mutant of thyroid transcription factor 1 (Titf1) (23), Spdef mRNA was markedly induced, supporting the concept that SPDEF might participate with TTF-1, directly or indirectly, to influence genetic programs regulating differentiation or function of the respiratory epithelium. Because of their overlapping 


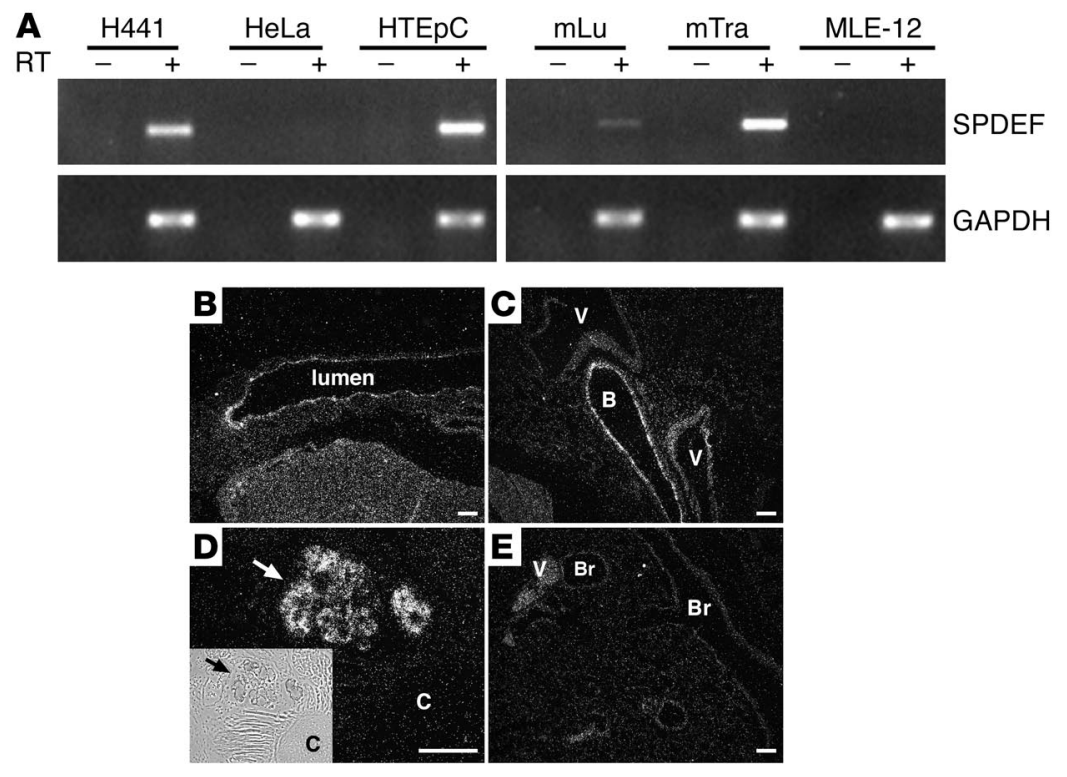

Figure 1

Spdef mRNA in mouse respiratory epithelial cells, trachea, and tracheal glands. (A) SPDEF and GAPDH expression was identified by RT-PCR using RNA extracts from human lung adenocarcinoma H441 cells, cervical adenocarcinoma HeLa cells, normal human tracheal epithelial HTEpC cells, SV40 large T immortalized mouse lung epithelial MLE-12 cells, mouse lung (mLu), and mouse trachea (mTra). Spdef mRNA was detected in H441 cells, HTEpC cells, and mouse lung and trachea, but not in HeLa or MLE-12 cells. PCR without RT (-) showed no product. (B-E) In situ hybridization for Spdef mRNA was performed on sections of trachea and tracheal glands (B and $\mathbf{D})$ and lung ( $\mathbf{C}$ and $\mathbf{E}$ ) in adult mice. Spdef mRNA was detected in the epithelium lining trachea, bronchi (B), and tracheal glands (arrows), but not in bronchioles $(\mathrm{Br})$ or blood vessels (V). Inset shows phase microscopy of the hybridized tracheal glands; original magnification, $\times 4$. Scale bars: $200 \mu \mathrm{m}$. C, cartilage.

expression in a subset of conducting airway epithelial cells, we sought to determine the role of SPDEF and its interaction, if any, with TTF-1 in airway epithelial cell differentiation.

In the present work, we demonstrated the expression of SPDEF in a subset of epithelial cells in tracheal glands and in the conducting airways of the fetal and adult mouse lung. SPDEF directly interacted with TTF-1 and influenced expression of a number of genes expressed in epithelial cells and conducting airways. IL-13- and STAT-6-dependent induction of SPDEF was observed in association with goblet cell hyperplasia. Expression of SPDEF in nonciliated airway epithelial cells (Clara cells) caused goblet cell hyperplasia, demonstrating its role in the regulation of airway epithelial differentiation.

\section{Results}

SPDEF is expressed in pulmonary epithelial cells. Mouse Spdef mRNA was detected in adult mouse lungs and trachea, but not in the MLE-12 cell line, an SV40 immortalized mouse lung epithelial cell line with characteristics of type II alveolar cells (Figure 1A). By in situ hybridization, Spdef mRNA was found to be present in subsets of respiratory epithelial cells in conducting airways of the mouse lung from E17.5 to adulthood (Figure 1 and Supplemental Figure 1; supplemental material available online with this article; doi:10.1172/JCI29176DS1). In lungs of adult mice, Spdef mRNA was readily detected in subsets of epithelial cells in trachea, bronchi, and epithelial cells of tracheal glands (Figure 1, B-E). Spdef mRNA was also present in H441, a human pulmonary adeno- carcinoma cell line, and HTEpC human tracheal epithelial cells, but not in HeLa cells (Figure 1A). In situ hybridization demonstrated the presence of Spdef mRNA in epithelial cells of the stomach, small intestine, caecum, colon, oviduct, dorsal and ventral prostate, coagulating gland, and seminal vesicles (Supplemental Figure 2), consistent with the previously reported distribution of Spdef mRNA in the adult mouse (25). An Spdef sense probe did not hybridize (Supplemental Figure 3).

Polyclonal antisera were produced against recombinant SPDEF that detected a single protein of approximately $37 \mathrm{kDa}$ by immunoblot. SPDEF antiserum-immunostained HeLa cells were transfected with a full-length mouse Spdef cDNA (Supplemental Figure 4). Consistent with our mRNA data, SPDEF was detected in epithelial cells lining the adult mouse trachea (Figure 2). Neither SPDEF staining nor mRNA was detected in bronchioles or alveoli (data not shown). Nuclear staining for SPDEF was observed in epithelial cells in trachea, bronchi, and tracheal glands, consistent with the distribution of Spdef mRNA detected by in situ hybridization (Figure 2). Sites of SPDEF expression in conducting airways overlapped with expression of TTF-1, SRY-box containing gene 17 (SOX17), FOXJ1, and SCGB1a1 (Figure 2). Spdef mRNA and protein were observed in prostate, oviduct, colon, and seminal vesicles (Supplemental Figures 2 and 5). In the adult lung, levels of staining intensity for SPDEF varied in nuclei of respiratory epithelial cells lining conducting airways (Figure 2, A and B). Relatively high levels of Spdef mRNA and immunostaining were observed in epithelial cells of tracheal glands (Figures 1 and 2). During development, Spdef mRNA was first detected in conducting airway epithelial cells of the fetal mouse lung at E17.5 (Supplemental Figure 1). Thereafter, Spdef was present in epithelial cells lining trachea and bronchi, but was not detected by either in situ hybridization or immunohistochemistry in peripheral bronchiolar and alveolar epithelial cells (Figures 1 and 2). Timing and sites of expression of SPDEF support its potential role in cell differentiation or gene expression in tracheal glands and proximal conducting airways, but not in peripheral bronchioles or alveolar epithelial cells in the mouse.

SPDEF interacts with TTF-1 and regulates gene expression in respiratory epithelial cells. Because SPDEF was selectively expressed in epithelial cells lining tracheal glands and proximal conducting airways, the ability of SPDEF to regulate potential transcriptional targets expressed at these cellular sites was assessed by transfection assays in vitro. Surfactant protein A is a host defense protein that is selectively expressed in epithelial cells of tracheal glands as well as bronchiolar and alveolar type II cells (26). SPDEF enhanced the activity of the Sftpa promoter in vitro (Figure 3A). Cotransfection of SPDEF with TTF-1 further activated the Sftpa promoter (Figure 3A). Potential SPDEF binding motifs GGAA/T (27) were identified in the promoter; however, repeated attempts to bind recombinant SPDEF or the DNA-binding domain of SPDEF to consensus SPDEF elements in the Sftpa promoter (aa 247-335) by EMSA were unsuccessful. Deletion of these potential SPDEF binding sites in the Sftpa gene 
A

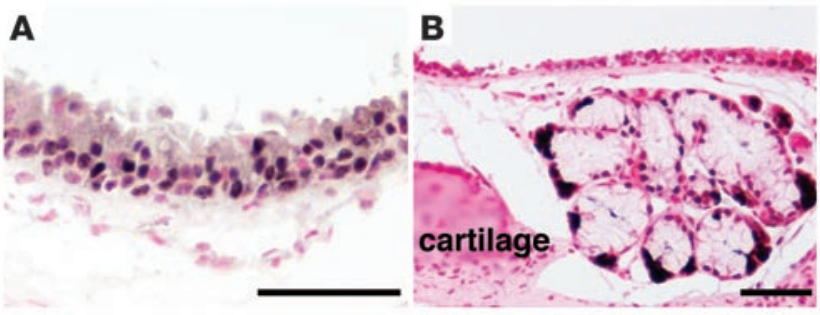

C

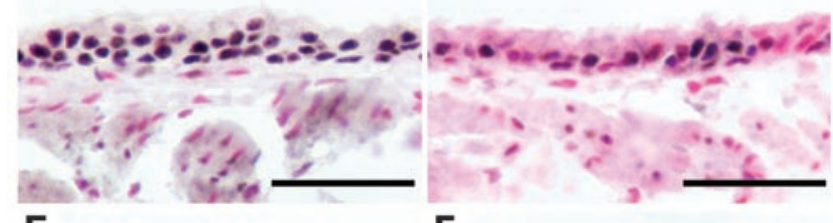

E

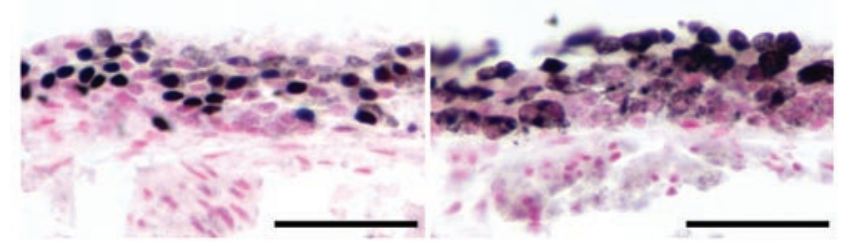

promoter did not inhibit its activation in the transfection assays (data not shown), suggesting that the effects of SPDEF on transcription may be mediated indirectly.

Because SPDEF was expressed in conducting airway epithelial cells, we tested whether SPDEF regulates the promoters of other genes expressed selectively in the proximal airways, including Foxj1, Sox17, Scgb1a1, and mucin 5, subtypes A and C, (Muc5A/C). SPDEF acted additively or synergistically with TTF- 1 on the pro-

\section{Figure 2}

SPDEF immunohistochemistry in mouse trachea and tracheal glands. Immunohistochemistry was performed on sections of adult lungs. (A and $\mathbf{B}$ ) SPDEF staining was detected in nuclei in epithelial cells lining the trachea (A) and tracheal glands (B). (C-F) Immunohistochemistry for SPDEF (A and B), TTF-1 (C), SOX17 (D), FOXJ1 (E), and SCGB1a1 (F) in tracheal epithelium. Scale bars: $50 \mu \mathrm{m}$.

moters of Foxj1, Scgb1a1, and Sox17 (Figure 3, B-D), but did not directly activate the $M U C 5 A / C$ gene promoter (data not shown). Again, we were unable to demonstrate direct binding of the recombinant SPDEF homeodomain or full-length SPDEF recombinant protein to the Foxj1 elements or to a previously reported consensus SPDEF binding site identified in the PSA gene by EMSA (24). Deletion and mutation of several potential SPDEF binding sites identified in the Foxj1 promoter did not block its activation by SPDEF (Supplemental Figure 6).

SPDEF interacts with the C-terminal domain of TTF-1. Because of the synergistic response to TTF-1 and SPDEF that we observed in several promoters, potential interactions between TTF- 1 and SPDEF were assessed by mammalian 2-hybrid assays in HeLa cells and by glutathione-S-transferase (GST) pulldown assays in vitro. TTF-1 and SPDEF directly interacted in both assays (Figure 4). Interactions of TTF- 1 with SPDEF were mediated by the C-terminal domain of TTF- 1 and did not require the TTF- 1 homeodomain, as assessed by both mammalian 2-hybrid assays and coimmunoprecipitation assays with GST-SPDEF (Figure 4). Thus, activation of target genes by SPDEF may be mediated, at least in part, by its interactions with TTF-1, known to bind and activate the promoters of a number of genes selectively expressed in the respiratory epithelium, including Sftpb, Sftpa, Sftpc, and Scgb1a1 (13-16).

Distinct effects of SPDEF and ETS variant gene 5. SPDEF enhanced expression of the promoters from the Sftpa, Foxj1, Sox17, and Scgb1a1 genes (Figure 3), but also activated the Sftpc gene, which is not normally expressed in conducting airway cells (data not shown).

\section{Figure 3}

SPDEF and TTF-1 activate gene transcription in vitro. Reporter assays were performed using plasmids expressing SPDEF and TTF-1 and reporter plasmids in which firefly luciferase gene (luc) is controlled by Sttpa (A), Foxj1 (B), Scgb1a1 (C), and Sox17 (D) gene promoters as described in Methods. SPDEF activated Sftpa, Foxj1, Scgb1a1, and Sox17 promoters in the presence and absence of TTF-1. Controls (Con) were cells transfected with the reported and empty expression plasmids. Assays were repeated in triplicate in at least 3 experiments with similar results. Values are mean $\pm \operatorname{SD}(n=3)$. $P$ values shown were obtained by ANOVA.
A
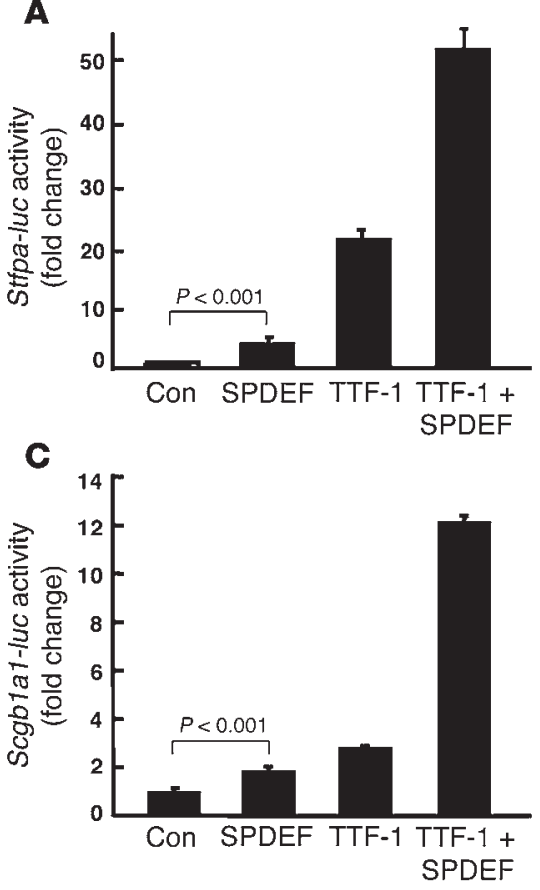

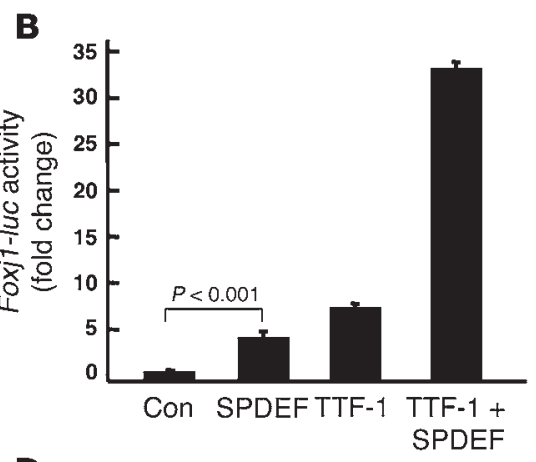

D

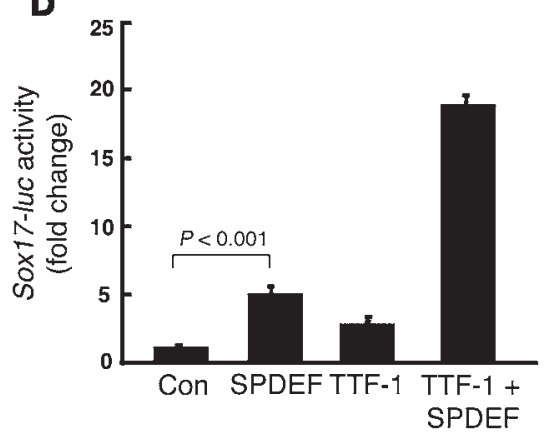


A

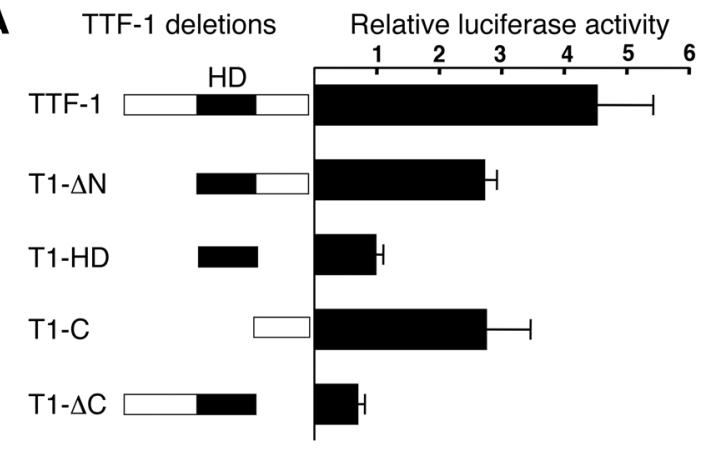

B

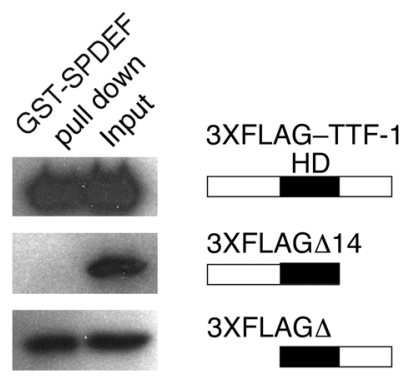

ETS variant gene 5 (ERM), a related member of the ETS family, is expressed in the distal epithelium during early lung development and is abundantly expressed in the peripheral lung saccules and in alveolar type II cells in the fetal and postnatal lung (28). ERM binds TTF- 1 and activates $S f t p c$ gene transcription in vitro (29). While SPDEF activated the Sftpa, Foxj1, Scgb1a1, and Sox17 promoters, ERM was inactive or less active (Figure 5). The distinct sites of expression of the ETS family members ERM and SPDEF and their selective influence on target genes may influence gene expression in a regionally specific manner.

SPDEF causes goblet cell hyperplasia in vivo. Because SPDEF was expressed in a subset of epithelial cells in the trachea, bronchi, and tracheal glands and stimulated transcriptional activity of genes normally expressed in proximal airway epithelial cells in vitro, its role was assessed in vivo. SPDEF was conditionally expressed under control of Clara cell secretory protein-reverse tetracycline transactivator (CCSP-rtTA; Figure 6A). Spdef transgene mRNA was not detected in lungs unless the mice were treated with doxycycline (Figure 6B and Figure 7). In situ hybridization and immunohistochemistry demonstrated the induction of Spdef mRNA and protein in subsets of cells in the respiratory epithelium lining conducting airways (Figure 6, D and $\mathrm{H}$ ) and alveoli (data not shown), consistent with the activity of the CCSP (Scgb1a1) promoter in this mouse line, which is known to selectively direct expression to Clara cells in the conducting airways $(30,31)$.

\section{Figure 5}

Comparison of SPDEF and ERM on target gene transcription. Reporter assays were performed using plasmids expressing SPDEF and ERM, an ETS family transcription factor also expressed in the lung. The plasmids were cotransfected with the firefly luciferase reporter plasmids under control of Sftpa (A), Foxj1 (B), Scgb1a1 (C), and Sox17 (D) gene promoters. While SPDEF activated the Sftpa, Foxj1, Scgb1a1, and Sox17 promoters, ERM was less active. Each assay was repeated in at least 3 experiments with similar results. Values are mean $\pm \mathrm{SD}(n=3)$. $P$ values shown were obtained by ANOVA.

\section{Figure 4}

SPDEF interacts with TTF-1 via the C-terminal domain of TTF-1. (A) Mammalian 2-hybrid assay was performed using the luciferase reporter pG5-luc and pACT and pBIND as described in Methods. Full-length TTF-1 and a series of TTF-1 deletion mutants (see Supplemental Table 1) were inserted to pBIND vector. Recombinant plasmids were cotransfected with the pG5-luc plasmids, and their activity was compared with that of cells transfected with pACT-SPDEF plus PBIND-TTF-1. Values are mean $\pm \operatorname{SD}(n=3)$. Assays were repeated 3 times with similar results. HD, homeodomain. (B) GST pulldown assays were performed with GST-SPDEF that was immobilized on glutathione-sepharose beads. Protein extracts were prepared from HeLa cells transiently transfected with the expression plasmids encoding for 3XFLAG-TTF-1, $3 X F L A G \Delta 14$, and $3 X F L A G \Delta 3$ as described in Methods. The extracts were incubated with GST or GST-SPDEF. Both GST and GST-SPDEF beads were washed several times before boiling, run on $10 \%$ SDSpolyacrylamide gels, and analyzed by immunoblot using a monoclonal antibody that recognizes the FLAG sequences.

SPDEF caused goblet cell differentiation in conducting airways of the transgenic mice (Figures 6-8). We observed similar results in 2 independent tetracycline response element 2-Spdef) (TRE2-Spdef) mouse lines, which were dependent upon doxycycline. Alcian blue staining (Figure 7, A and B) and immunostaining for MUC5A/C (Figure 7, C and D) were increased at the sites of SPDEF expression in the trachea and bronchi and in peripheral airways, including smaller bronchioles that normally lack goblet cells. Morphometric analysis demonstrated that SPDEF induced goblet cell hyperplasia in large (cartilagenous), medium-sized (central), and lateral (distal) airways, but not in terminal bronchioles or in the alveoli (Figure 8). There was a significant increase in the amount of Alcian blue staining found in the doxycycline-treated group compared with the untreated group $(P=0.004$; Kruskal-Wallis 1 -way ANOVA on ranks). Pairwise comparisons of each airway category revealed that the extent of Alcian blue staining in large, proximal, hilar airways increased significantly in the doxycycline-treated group compared with the untreated group $(P=0.026$; Figure 8$)$. The extent of Alcian blue staining along the airway correlated with staining for SPDEF

A

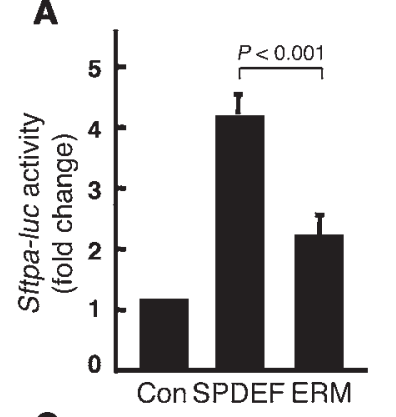

C

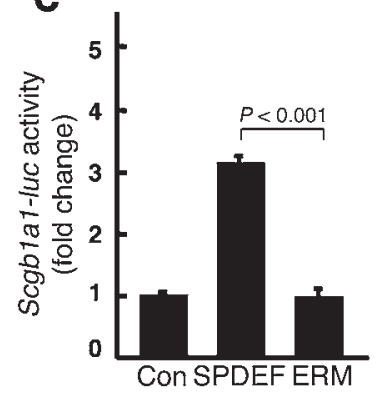

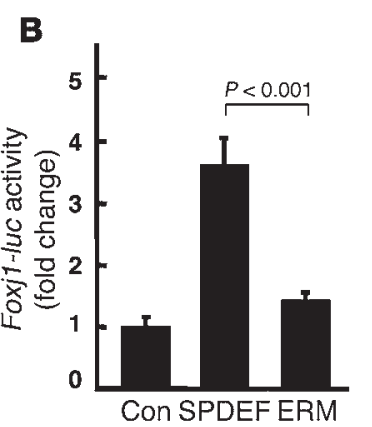

D

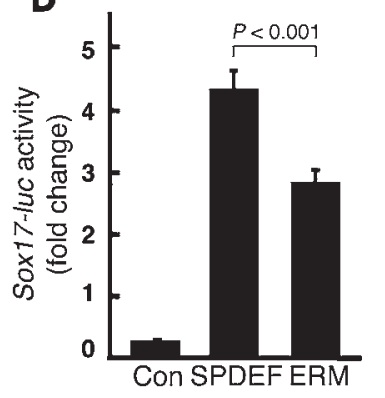


A

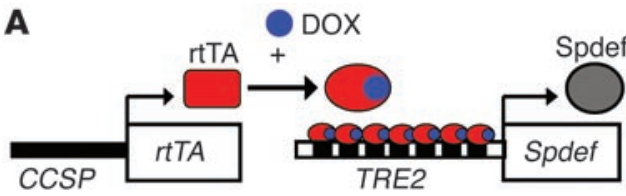

B
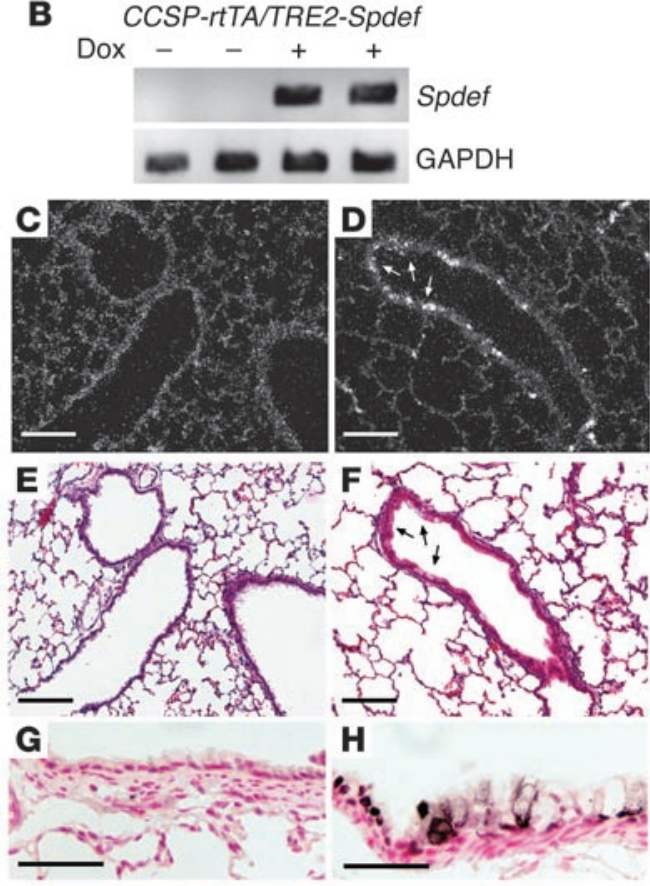

in larger conducting airways, but was not significant in the more distal bronchioles, acinar ducts, or alveoli (Figure 8). Goblet cell hyperplasia occurred in the absence of inflammation, leukocytic infiltration, or altered expression of TGF- $\alpha$, heparin-binding EGF (HB-EGF), IL-4, IL-6, and IL-13 mRNAs (Supplemental Figure 7). CCSP staining was decreased in regions lined by goblet cells (Figure 7, E and F), whereas the staining pattern for FOXJ1, a ciliated cell marker, was not altered (Figure 9, A and B). Because the CCSP-rtTA driven Spdef transgene is expressed selectively in Clara cells, the paucity of CCSP staining and the presence of goblet cell hyperplasia that we observed in vivo are consistent with a cell-autonomous effect of SPDEF on the differentiation of Clara cells into goblet cells. Since loss of FOXA2 was previously shown to cause goblet cell differentiation (9), we assessed the effect of SPDEF on FOXA2 expression. FOXA2 staining was absent at sites of goblet cell hyperplasia induced by SPDEF (Figure 9, C and D). Phosphorylated histone H3 ( $\mathrm{pH} 3$ ) staining was used to identify proliferating cells. The ectopic goblet cells did not stain for $\mathrm{pH}$,

\section{Figure 7}

Expression of SPDEF caused goblet cell hyperplasia in the conducting airways. CCSP-rtTA/TRE2-Spdef mice were maintained with (B, $\mathbf{D}$, and $\mathbf{F}$ ) or without (A, C, and $\mathbf{E})$ doxycycline from E0 to postnatal day 14. Lung sections were stained with Alcian blue (A and $\mathbf{B})$ or by immunohistochemistry for MUC5A/C (C and D) and CCSP (E and F). Increased Alcian blue and MUC5A/C staining was readily detected in the conducting airways of mice in the presence (B and $\mathbf{D})$, but not in the absence (A and $\mathbf{C}$ ), of doxycycline. Expression of SPDEF caused decreased CCSP staining (F) compared with controls without doxycycline (E). Scale bars: $100 \mu \mathrm{m}$.

\section{Figure 6}

Conditional expression of SPDEF in vivo. (A) Construct and strategy used to express SPDEF in Clara cells in the conducting airway. (B) RT-PCR using primers that selectively detect transgenic Spdef mRNA revealed that transgene-specific Spdef mRNA was increased in whole lung in the presence, but not the absence, of doxycycline (Dox). GAPDH expression was detected as an internal control. (C-H) In situ hybridization ( $\mathbf{C}$ and $\mathbf{D}$ ) and immunostaining ( $\mathbf{G}$ and $\mathbf{H}$ ) were performed to detect the expression of Spdef mRNA and protein in conducting airways and lung parenchyma in the absence (C, E, and $\mathbf{G})$ and the presence $(\mathbf{D}, \mathbf{F}$, and $\mathbf{H})$ of doxycycline. (E and $\mathbf{F})$ Serial sections from $\mathbf{C}$ and $\mathbf{D}$ were stained with hematoxylin and eosin. Spdef mRNA and protein were detected at the sites of goblet cell morphology in the conducting airways of CCSP-rtTA/TRE2-Spdef mice treated with doxycycline (arrows), but were not detected in the bronchiolar epithelium of the transgenic mice without doxycycline. Scale bars: $200 \mu \mathrm{m}$ (C-F); $50 \mu \mathrm{m}$ ( $\mathbf{G}$ and $\mathbf{H})$.

supporting the concept that expression of SPDEF in the airway epithelium influenced cell differentiation rather than proliferation (Supplemental Figure 8).

Induction of SPDEF expression in mouse models of goblet cell hyperplasia. Increased expression of either IL-4 or IL-13 (32-35) and allergen challenge (36) cause goblet cell hyperplasia in vivo. We tested whether increased SPDEF expression is associated with goblet cell hyperplasia in mice expressing IL-13 in Clara cells under conditional control of doxycycline. Increased SPDEF staining and mRNA were associated with goblet cell hyperplasia in conducting airways of adult mice as assessed by RT-PCR, in situ hybridization, and immunostaining (Figure 10). SPDEF staining was observed in both the cytoplasm and the nuclei of epithelial cells in conducting airways. Likewise, intratracheal IL-13 treatment caused goblet cell hyperplasia in association with increased SPDEF staining in control but not in Stat-6-/- mice (Figure 11, A and B). Goblet cell hyperplasia and increased SPDEF staining were observed following

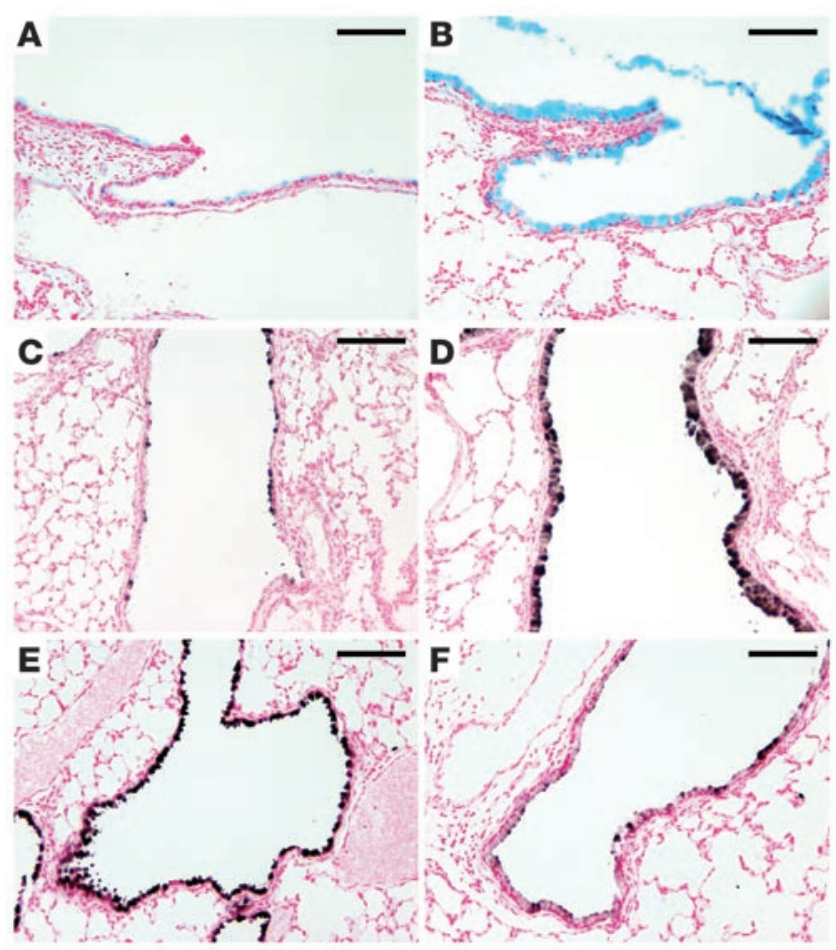


A
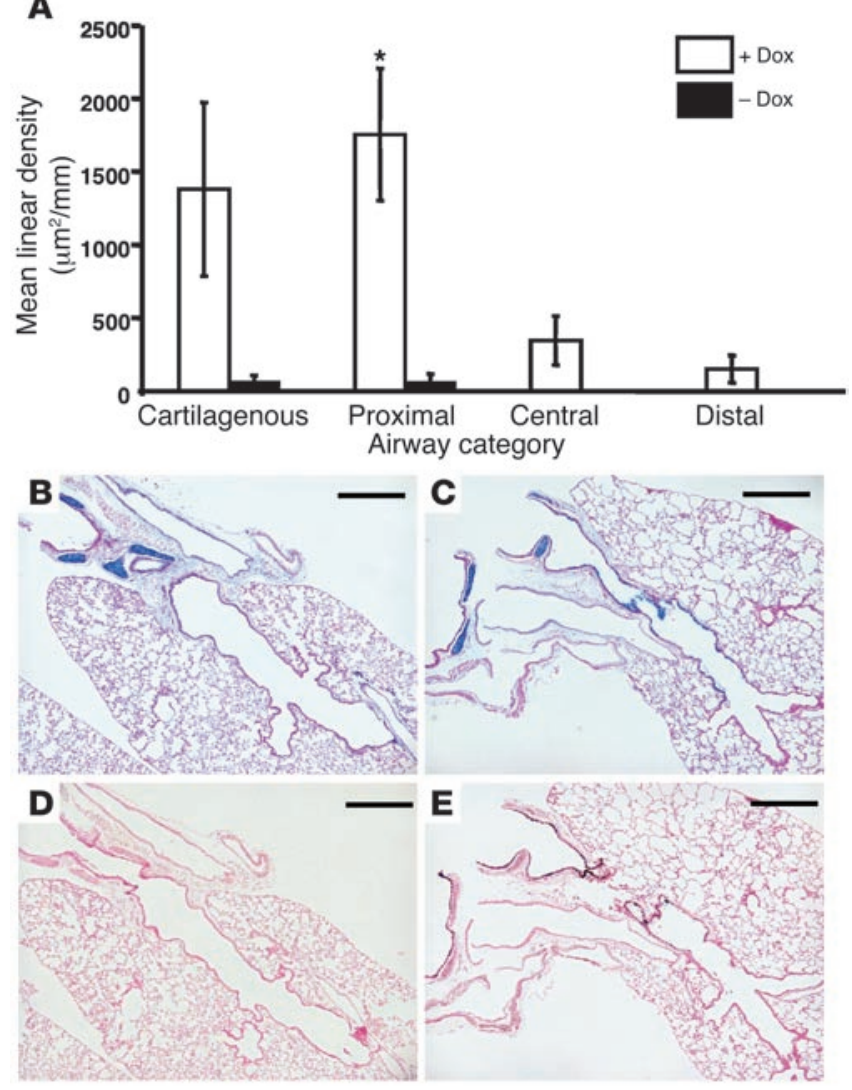

repeated intratracheal administration of dust mite allergen to wildtype mice but was not observed in treated $I L-13^{-/-}$mice (Figure 11, C and D). Thus, IL-13 and allergen exposure increased Spdef mRNA and staining, extending expression in smaller conducting airways.

\section{Discussion}

The trachea and bronchi of the mouse are lined by a complex pseudostratified epithelium that is composed of ciliated, nonciliated (Clara and goblet), and basal cells. When mice are chronically exposed to allergens or toxicants, goblet cell hyperplasia is frequently observed. The present study demonstrated expression of SPDEF in epithelial cells lining the trachea, bronchi, and tracheal glands. The temporal/spatial pattern of expression of SPDEF during development, and the finding that SPDEF activated the Sftpa, Foxj1, Scgb1a1, and Sox17 promoters, support the concept that SPDEF regulates cell differentiation and gene expression in airway epithelial cells or their precursors during lung morphogenesis. The observed mucous cell hyperplasia and increased mucous production caused by expression of SPDEF in Clara cells in the conducting airway cells supports the concept that SPDEF functions in a cellautonomous manner to cause goblet cell hyperplasia. Dust mite exposure and the presence of IL-13 induced SPDEF expression and caused goblet cell hyperplasia, consistent with a role for SPDEF in goblet cell differentiation caused by Th2 cytokines and allergens.

SPDEF interacts with TTF-1. SPDEF and TTF-1 are coexpressed in subsets of proximal airway epithelial cells. The activity of SPDEF on expression of several target genes was enhanced by TTF-1, supporting a role for their interactions in the regulation of cell differentiation and gene expression. SPDEF bound to TTF-1 in mam-

\section{Figure 8}

Morphometric analysis of goblet cell hyperplasia. (A) Alcian blue staining $\left(\mu \mathrm{m}^{2} / \mathrm{mm}\right)$ was increased significantly in CCSP-rtTA/TRE2-Spdef mice exposed to doxycycline compared with unexposed mice $(P=0.004$; Kruskal-Wallis 1 -way ANOVA on ranks). Staining was increased in all 4 categories of airways: cartilagenous, proximal (noncartilagenous), central, and distal. Pairwise comparisons of the data for the 4 different airway categories demonstrated that the increase in Alcian blue staining was most significant in the proximal airways of the doxycycline-exposed double-transgenic mice. ${ }^{*} P=0.026$; Mann-Whitney rank-sum test. (B) Alcian blue staining was observed only in airways associated with cartilage, not in the epithelia of noncartilagenous airways, in double-transgenic mice without doxycycline. (C) Alcian blue staining was observed in epithelial cells lining these regions conducting airways in the presence of doxycycline. (D) Minimal SPDEF staining was observed in control mice at this antibody dilution. (E) Increased staining for SPDEF was observed in conducting airways of doxycyclineexposed double-transgenic mice. Scale bars: $500 \mu \mathrm{m}$.

malian 2-hybrid and pulldown assays, a finding that supports their physical interaction. These protein-protein interactions may represent a conserved relationship between SPDEF and other Nkx homeodomain factors. ERM, an ETS factor expressed in peripheral lung tubules of the developing lung, binds TTF-1 and subsequently activates the $S f t p c$ gene promoter (29). In the present study, ERM was found to be less active or inactive in airway epithelial cell gene promoters (Sox17, Scgb1a, Foxj1, and Sftpa). Thus, the distinct cellular sites of expression and activities of ERM and SPDEF may determine gene expression along the cephalocaudal axis of the lung. The observed interactions among Nkx and ETS family members are consistent with previous findings in prostatic carcinoma cells, in which Nkx-3.1 interacted with SPDEF to negatively modulate gene expression in vitro (37). As the potential

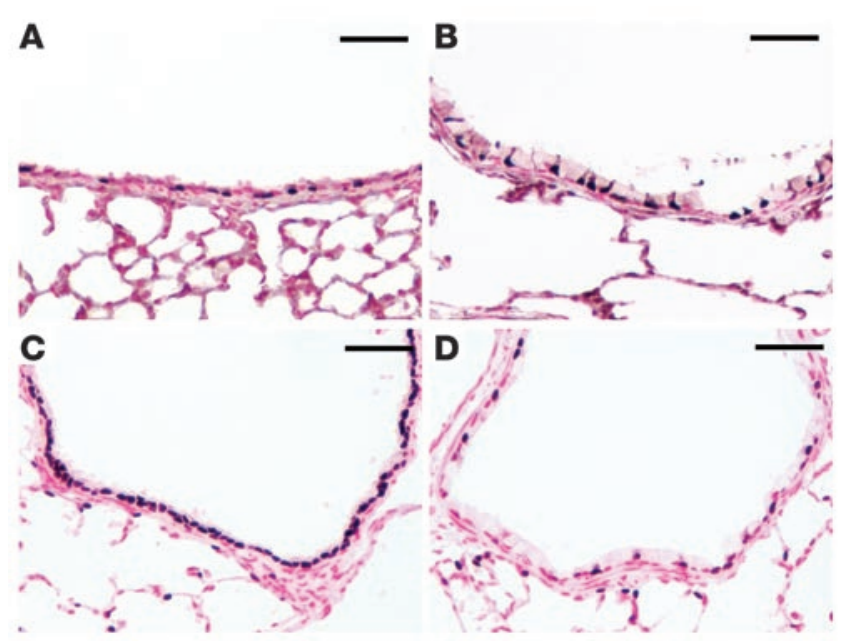

Figure 9

FOXJ1 and loss of FOXA2 staining in lungs of CCSP-rtTA/TRE2-Spdef transgenic mice. Immunohistochemistry for FOXJ1 (A and $\mathbf{B}$ ) and FOXA2 (C and D) was performed on the lung sections of control mice $(\mathbf{A}$ and $\mathbf{C})$ and transgenic mice expressing SPDEF (B and $\mathbf{D})$. The normal staining pattern of FOXJ1, a ciliated cell marker, was unaltered by expression of SPDEF (A and B). FOXA2 staining was observed throughout the epithelium in control mice (C). FOXA2 was not detected in the goblet cells lining the conducting airways of the transgenic mice, but persisted in ciliated cells (D). Scale bars: $50 \mu \mathrm{m}$. 
A
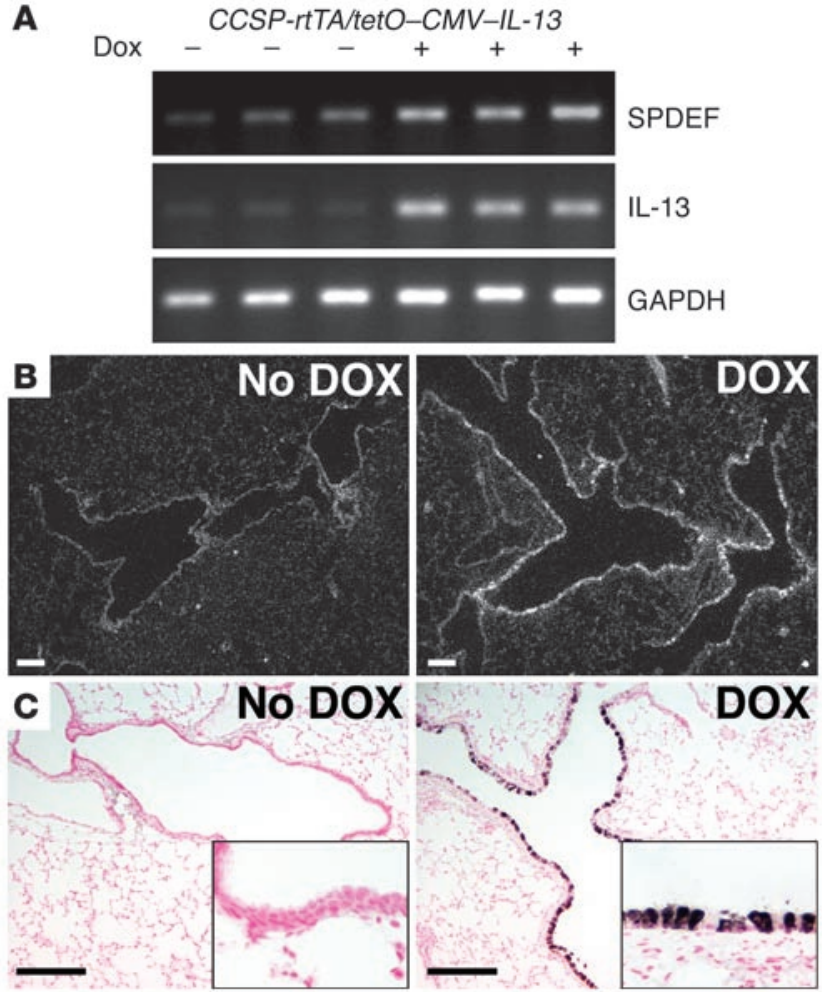

binding sites of SPDEF and those previously identified for TTF-1 in $S f t p a$ and $S c g b 1 a 1$ promoters are located in close proximity, these transcription factors may directly or indirectly interact to modulate gene expression. In the present study, SPDEF activated the promoters of a number of genes expressed in the conducting airways, acting synergistically or additively with TTF-1 on target genes. Although SPDEF was shown to bind consensus elements in the PSA gene (24), we were repeatedly unable to demonstrate direct binding of recombinant SPDEF or the SPDEF homeodomain to potential Spdef elements from the PSA, Foxj1, or Sftpa genes in vitro. Likewise, deletion of SPDEF consensus elements in the Sftpa and Foxj1 genes failed to inhibit its activity on gene expression in vivo. Thus, it remains unclear whether transcriptional effects of SPDEF are mediated by its direct binding to target genes or by its binding to TTF- 1 or other factors that bind and activate transcriptional targets. Alternatively, SPDEF may alter the expression of other factors that influence target gene expression. Since TTF-1 and SPDEF are coexpressed in various respiratory epithelial cells, the specificity of their transcriptional activity may be determined by their direct interaction, by interactions with other transcription factors and coactivators, or by the differences in their expression levels in various cell types lining the airways. The finding that SPDEF inhibited CCSP expression in vivo contrasts with its stimulatory effect on the Scgbla1 promoter in vitro. However, expression of SPDEF in Clara cells caused goblet cell differentiation, resulting in loss of Clara cells.

Role of SPDEF in differentiation of goblet cells and pathogenesis of goblet cell hyperplasia. SPDEF expression was restricted to epithelial cells lining the trachea, bronchi, and tracheal glands, where mucus-producing cells, including goblet cells, are normally found. Expression of SPDEF in Clara cells in the respiratory epithelium of adult transgenic mice was sufficient to cause goblet cell differentiation

\section{Figure 10}

IL-13 induces expression of SPDEF. At 5 weeks of age, CCSP-rtTA/ tetO-CMV-IL-13 mice were maintained with or without doxycycline for 1 week. (A) RT-PCR for SPDEF was performed using total RNA from the transgenic mice. Spdef mRNA was increased in the transgenic mice treated with doxycycline, while GAPDH was unchanged. (B and C) In situ hybridization (B) and SPDEF immunostaining (C) were performed on lung sections from the transgenic mice. Spdef mRNA was induced in the conducting airways of the transgenic mice treated with doxycycline but was not detected in the untreated mice (B). SPDEF staining was detected in the conducting airways of the transgenic mice treated with doxycycline $(\mathbf{C})$, consistent with the sites of Spdef mRNA expression. SPDEF was not detected in the absence of doxycycline under these conditions. Insets represent higher magnification views of these airways; original magnification, $\times 20$. Scale bars: $200 \mu \mathrm{m}$.

in large- and medium-sized conducting airways but not in cells lining bronchoalveolar ducts or the alveoli. This finding suggests that the regional specific goblet cell differentiation caused by SPDEF may depend upon other factors that are selectively expressed in the larger airways, or that factors expressed in the peripheral lung inhibit its activity. Taken together, the results of the present study link SPDEF to goblet cell hyperplasia, but it is unclear whether SPDEF is required for goblet cell differentiation in the lung. Relevant to the present findings, expression of SPDEF was markedly reduced in the intestines of $\mathrm{Gfi1}^{-/-}$mice, in which the number of goblet cells was decreased (38), suggesting a relationship between SPDEF and goblet cell differentiation.

Expression of SPDEF in Clara cells of the postnatal mouse lung reduced expression of CCSP. This finding contrasts with our in vitro findings that SPDEF induced the Scgb1a1 promoter in HeLa cells and H441 cells in vitro. Since the expression of SPDEF in

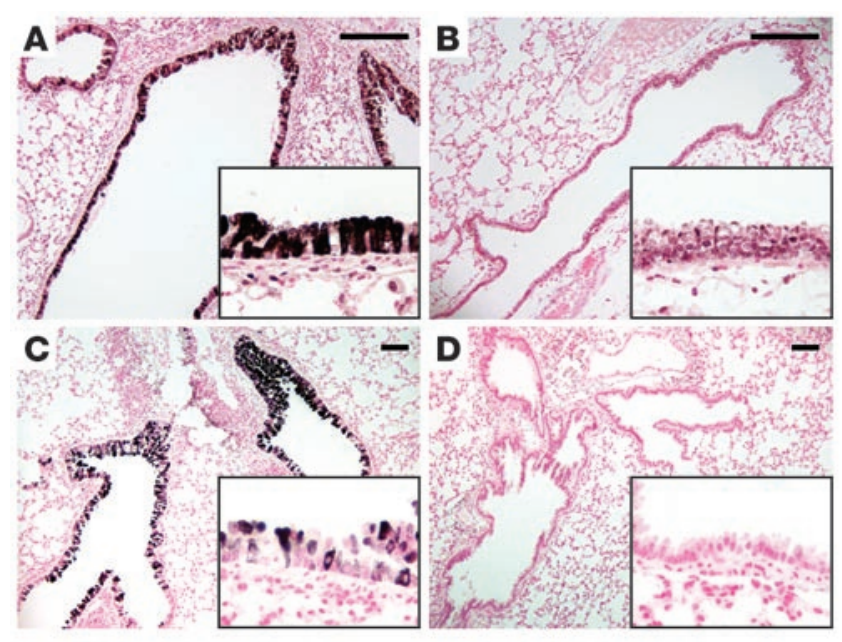

Figure 11

IL-13 and dust mite allergen induce SPDEF and cause goblet cell hyperplasia. (A and B) Immunohistochemistry for SPDEF was performed on lung sections of control $(\mathbf{A})$ and Stat- $6^{-/-}(\mathbf{B})$ mice that were treated intratracheally with IL-13. SPDEF staining was increased at sites of goblet cell hyperplasia, but absent in conducting airways of Stat-6 $6^{-/}$mice. (C and D) SPDEF was increased in association with goblet cell hyperplasia caused by intratracheal exposure to house dust mite allergens in wildtype mice (C), but not in the conducting airways of exposed $/ L-13^{-/-}$mice (D). Insets for A-D represent higher magnification views of these airways; original magnification, $\times 20$. Scale bars: $200 \mu \mathrm{m}$. 
Clara cells caused their differentiation into goblet cells, decreased CCSP staining may be related to inhibition of Clara cell differentiation, rather than to its effect on the Scgbla1 gene promoter. Alternatively, the effects of SPDEF on CCSP expression may be cell type specific. Goblet cell differentiation in the Spdef transgenic mice was not accompanied by the induction of inflammatory cytokines or growth factors known to cause goblet cell hyperplasia. These findings suggest that SPDEF played a cell-autonomous role in the transdifferentiation of nonciliated airway epithelial cells into goblet cells. SPDEF was induced in the airway epithelium in mouse models associated with goblet cell hyperplasia. Allergen and IL-13 exposure caused marked goblet cell hyperplasia associated with MUC5A/C expression, mucous hyperproduction, and increased SPDEF expression. STAT- 6 was required for both goblet cell hyperplasia and enhanced expression of SPDEF, indicating that SPDEF may play an important role in the transcriptional regulation of allergic responses in the respiratory epithelium. Whether increased mucin production is mediated by effects of SPDEF on mucin gene expression or by a more complex effect on cell differentiation is unclear. Since FOXA2 is required for maintenance of normal respiratory epithelial cell differentiation, the inhibitory effect of SPDEF on FOXA2 expression may contribute to or cause the goblet cell hyperplasia induced by SPDEF in vivo.

In summary, we detected SPDEF in proximal conducting airway epithelial cells and in tracheal glands in the normal mouse. SPDEF bound TTF-1 and coactivated Foxj1, Sox17, Scgb1a1, and Sftpa gene promoters in vitro. Increased expression of SPDEF in Clara cells in the conducting airways of transgenic mice caused goblet cell hyperplasia. Allergen and Th2 cytokine exposure induced SPDEF expression and caused goblet cell hyperplasia in the conducting airways in vivo. These findings support the concept that SPDEF plays an important role in differentiation and gene expression in proximal airway epithelial cells and identifies a transcriptional mechanism linking Th2 cytokine activity with the transdifferentiation of Clara cells to goblet cells.

\section{Methods}

Generation of plasmids and antibodies. cDNA was prepared by reverse transcription using total RNA isolated from cultured cells of trachea and lung parenchyma. SPDEF CDNA containing the entire open reading frame was amplified from E18 fetal lung using PCR primers (forward, 5'-CTTCTGACAGCAGGCGGCTAAC-3'; reverse, 5'-GACTGGATGCACAAATTGGTAGACAAG-3') based on the sequence of GenBank accession number NM013891 (25). The amplified PCR product was cloned into pcDNA 3.1/ V5-His-Topo (Invitrogen). The GST-SPDEF cloned insert was sequenced and confirmed with the database sequence. TTF- 1 and the TTF- 1 deletion plasmid constructs, including $\Delta 3$ TTF- 1 ( $\mathrm{N}$-terminal deletion) and $\Delta 14$ TTF-1 (C-terminal deletion), were previously described and kindly provided by M. deFelice (Stazion Zoological, Naples, Italy; refs. 23, 39). TTF-1 plasmids used for mammalian 2-hybrid assays (Supplemental Table 1) were as previously described (17). The reporter plasmids, Sftpa-luc (1.1-kb mouse Sftpa promoter-pGL3), Scgb1a1-luc (2.3-kb rat Scgb1a1 promoterpGL2), Sftpc-luc (4.8-kb mouse Sftpc promoter-pGL2), MUC5A/C-luc (4.0-kb human MUC5A/C promoter-pGL2), Foxj1-luc (1.1-kb mouse Foxj1 promoter-pGL3), and pG5-luc express the luciferase gene under control of each promoter $(19,40-42)$. For 0.62 and 0.25 Foxj1-luc plasmids, $1.1-\mathrm{kb}$ Foxj1-luc was cut at Xmn1 and Msc1 sites and ligated, respectively. For mutations of consensus SPDEF binding sites, 143-bp oligonucleotides containing each mutation were introduced by using the unique Bsu36I restriction site ( 3 ' end position -231 to -222 from ATG) within the 0.25
Foxj1-luc and Nhe1 site. The wild-type sequence was removed by digesting 0.25 Foxj1-luc plasmid with Nhe1 and Bsu36I and replaced with the oligonucleotides that were annealed and then digested with Nhe1 and Bsu36I. Sox17-luc was constructed by cloning the 3.6-kb 5' untranslated region (UTR) of the mouse Sox17 gene in pGL3B (Promega). The Sox17 UTR was amplified from the tail genomic DNA of adult FVB/N mice using Expand High Fidelity kit (Roche) and the following primers: forward, 5'-TTGACGCGTGTTATCTTAGAGTCCGCCG-3', reverse, 5' -AAACTCGAGATGGCTCTCCAGACCGAC-3'. The PCR fragments were cloned into pGL3 Basic using MluI and XhoI sites (shown in bold) and sequenced. Guinea pig polyclonal antibodies were generated against a fragment of recombinant SPDEF protein (aa 3-243) fused to a $6 \times$ His-tag. A partial cDNA encoding aa 3-243 of Spdef was amplified and cloned into an E. coli PTrcHis-TOPO expression vector (Invitrogen). The recombinant SPDEF peptide was expressed in E. coli and purified using nickel chromatography affinity according to the manufacturer's instructions (Novagen).

$R T-P C R$. Total RNA was prepared from a mouse lung epithelial cell line (MLE-12), a human pulmonary adenocarcinoma cell line (H441), and human tracheal epithelial cells (HTEpc; Cell Applications Inc.) using TRIzOL according to the manufacturer's protocol (Invitrogen). For RT-PCR, CDNA was generated by reverse transcription using the total RNA. A 240bp fragment of human $S P D E F$ was amplified using primers (forward, $5^{\prime}$ TGTCCGCCTTCTACCTCTCCTAC-3'; reverse, 5' -CGATGTCCTTGAGCACTTCGC-3'). A 407-bp fragment of mouse Spdef was amplified using primers (forward, 5'-GTTGCCTGCTACTGTTCCCAGATG-3'; reverse, 5'AAAGCCACTTCTGCACGTTACCAG-3'). A 238-bp fragment of GAPDH was amplified using primers (forward, 5'-CTTCACCACCATGGAGAAGGC-3'; reverse, 5' -GGCATGGACTGTGGTCATGAG-3'). The PCR products were resolved by gel electrophoresis on $1.5 \%$ agarose gels containing ethidium bromide. RT-PCR for Spdef, IL-13, IL-4, IL-6, TGF- $\alpha$, and HB-EGF was performed using $2 \mu \mathrm{g}$ total RNA from CCSP-rtTA/TRE2-Spdef mice treated with or without doxycycline ( $n=6$ per group) using the primers described in Supplemental Table 2. PCR products were identified on the gel-electrophoresis and scanned for quantification using ImageQuant (GE HealthCare). Lack of DNA contamination was verified by RT-PCR with presence or absence of RT.

Immunobistochemistry. Lung tissue was dissected from fetal and postnatal mice, fixed with $4 \%$ paraformaldehyde in PBS, dehydrated, and embedded in paraffin according to standard methods (43). Immunostaining for SPDEF was performed essentially as described previously (44). Anti-SPDEF polyclonal guinea pig antibody was produced and used at a dilution of 1:10,000. FOXJ1, SOX17, FOXA2, CCSP (SCGB1a1), MUC5A/C, and TTF-1 antibodies and immunohistochemistry have been described previously $(8,9,42)$.

Morphometric analysis. Double-transgenic mice were maintained with $(n=4)$ or without $(n=3)$ doxycycline treatment from E0.5 to 6 weeks of age, and their lungs were inflation-fixed with $4 \%$ paraformaldehyde in PBS. Lungs were excised from the thorax, and each lobe was bisected and embedded in 1 block with the cut (i.e., flat) side down for sampling purposes $(n=4-5$ lobes per block). Paraffin sections were cut at 4-6 $\mu \mathrm{m}$ and numbered sequentially for histochemical, immunohistochemical, and quantitative analyses. Slides with sections demonstrating the greatest number and variety of proximal and distal airways were selected for staining with the Alcian blue technique ( $n=2$ sections per lobe, $4-5$ lobes per block for each animal). Consecutive sections were then immunostained for SPDEF and MUC5A/C expression. The conducting airways in each section were classified into 4 different categories: cartilagenous, airways with associated cartilage; proximal, large, hilar airways with associated pulmonary vein and artery; central, medium-sized airways; and distal, smaller airways, including the lateral branches and terminal airways. Digital images of each airway were acquired with a Nikon 90I wide field microscope (original magnification, $\times 4$ ) and imported into 
MetaMorph imaging software version 6.2 (Universal Imaging Corp.). Alcian blue staining was quantified by tracing the area (in $\mu \mathrm{m}^{2}$ ) of epithelial/cellular staining in each airway. Area measurements for this parameter reflected both length and height of airway epithelia positive for Alcian blue staining. The amount of airway epithelia surveyed was quantified by tracing the perimeter and/or longitudinal length (in $\mathrm{mm}$ ) of each airway along the basement membrane. Area measurements for Alcian blue staining were then totaled and normalized to total length of epithelia surveyed to give a density value $\left(\mu \mathrm{m}^{2} / \mathrm{mm}\right)$ for each airway. Density values from each animal in the doxycycline treated $(n=4)$ or untreated $(n=3)$ groups were then combined in order to obtain the mean density for each airway category in the 2 different treatment groups. Data were graphed as mean \pm SEM density. Kruskal-Wallis 1-way ANOVA on the ranks (SigmaStat version 2.0; Systat Software Inc.) was used to determine overall statistical significance for Alcian blue staining between the 2 treatment groups. Data in the cartilagenous, proximal, and distal airway categories passed both the normality and equal variance tests; therefore, 1-tailed Student's $t$ test of the means (SigmaStat version 2.0; Systat Software Inc.) was used for these pairwise comparisons. Data in the central airway category did not pass either the normality or the equal variance tests; therefore, Mann-Whitney rank sum test was used for statistical comparison.

In situ hybridization. Riboprobes were synthesized from a 440-bp cDNA template for Spdef containing 50 nucleotides of the 5' UTR and 389 nucleotides of coding sequences, subcloned into a pGEM $3 Z$ transcription vector (Promega). Riboprobes were synthesized using T7 (antisense) and SP6 (sense) polymerases and reagents contained in a commercial transcription kit (Riboprobe In Vitro Transcription Systems; Promega) and labeled with $1,000 \mathrm{Ci} / \mathrm{mmol}$ of [35-S]-UTP and $800 \mathrm{Ci} / \mathrm{mmol}$ of [35-S]-CTP (Amersham Biosciences). Single-stranded transcripts were separated from unincorporated nucleotides by column chromatography, precipitated in ammonium acetate and ethanol, and reconstituted in $200 \mathrm{mM}$ DTT. For hybridization, the riboprobes were diluted in hybridization buffer to a final concentration of $5 \times 10^{4} \mathrm{cpm} / \mu \mathrm{l}$. Pretreatment, hybridization of tissue sections overnight $\left(58^{\circ} \mathrm{C}\right)$, and posthybridization high-stringency washes were performed as described previously (45). Sections were dehydrated, dipped in Ilford K5 nuclear research emulsion (Polysciences Inc.), exposed for 2-6 weeks, and developed with Kodak D19 developer (Eastman Kodak). The sections were then examined and photographed under dark-field illumination with a Nikon Microphot FXA wide-field photomicroscope.

Transfection assays. HeLa cells were maintained as previously described (17). Cells were seeded at $1 \times 10^{5}$ per well in 6 - or 12 -well plates and transfected with the plasmids using Lipofectamine 2000 (Invitrogen) or Effectene (QIAGEN) according to the manufacturers' instructions. The amount of transfected DNA was kept constant by addition of corresponding amounts of the backbone plasmid. pCMV- $\beta$-galactosidase or pRL-TK encoding Renillar luciferase was also transfected. After 36-48 hours of incubation, lysates were assayed for $\beta$-galactosidase and luciferase activities (Promega). Light units were assayed by luminometry (Monolight 3010; Analytical Luminescence Laboratory). Firefly luciferase activities (in relative light units) were normalized to $\beta$-galactosidase or Renillar luciferase activity. All assays were performed in triplicate in at least 3 separate experiments.

Conditional expression of SPDEF in the mouse lung. Mice expressing SPDEF were generated by cloning the full-length mouse SPDEF coding sequence (including Kozak sequences) at the SalI and XbaI sites in the PTRE2 vector (Clontech). Primers were synthesized for the SPDEF coding sequence with SalI and XbaI enzyme restriction sites shown in bold (forward, 5 '-CCCGGGGTCGACCGCAGCATGGGCAGTGCCAGCCCAGG-3'; reverse, 5'CCCGGgtCTAgAtCAgACTGGatgCACAAATTGGTAG-3'). Amplification of PCR products was performed as follows: denaturation at $94^{\circ} \mathrm{C}$ for 2 minutes; 35 cycles of denaturation at $94^{\circ} \mathrm{C}$ for 30 seconds, annealing at $60^{\circ} \mathrm{C}$ for 30 seconds, and extension at $72^{\circ} \mathrm{C}$ for 1 minute, followed by a 7 -minute extension at $72^{\circ} \mathrm{C}$. Following amplification, PCR products were digested with SalI and XbaI and cloned into PTRE2 digested with SalI and XbaI. pTRE2-SPDEF clones were confirmed by DNA sequencing of both strands. pTRE2-SPDEF was digested with AatII and SapI, and the resulting fragment containing SPDEF was microinjected into FVBN embryos by the Children's Hospital Research Foundation Transgenic Animal Facility. Founder mice were identified by PCR. Briefly, transgenic mice were identified using PCR primers specific for the TRE2-Spdef transgene (forward SPDEF coding sequence, 5'-TGAACATCACAGCAGACCC-3'; reverse PTRE2 vector sequence, 5'-TCTTCCCATTCTAAACAACACC-3'). Amplification of PCR products was performed as follows: denaturation at $94^{\circ} \mathrm{C}$ for 2 minutes; 35 cycles of denaturation at $94^{\circ} \mathrm{C}$ for 30 seconds, annealing at $60^{\circ} \mathrm{C}$ for 30 seconds, and extension at $72^{\circ} \mathrm{C}$ for 30 seconds, followed by a 7 -minute extension at $72^{\circ} \mathrm{C}$. Four founder mice were identified as containing SPDEF and PTRE2 sequences. For lung-specific, doxycycline-induced recombination, the CCSP-rtTA transgene was used $(30,31)$. CCSP-rtTA mice were bred with each of the 4 founding mice containing SPDEF. Offspring were identified using PCR primers specific for the CCSP-rtTA and TRE2Spdef transgenes as above $(30,31)$.

Mammalian 2-bybrid system assays. SPDEF, TTF-1, and TTF-1 mutant CDNAs were generated by PCR and cloned into the vectors PACT and pBIND in the mammalian 2-hybrid system kit (Promega) between BamHI and XbaI sites (Supplemental Table 1). HeLa cells were plated in 6-well plates at a density of $5 \times 10^{4} 24$ hours before cells were transfected with Effectene transfection reagent (QIAGEN). Cells were transfected with pG5luc and pACT-SPDEF, and PBIND-TTF-1, and PBIND-TTF-1 mutants using $0.04 \mathrm{pM}$ of each vector per well. Forty-eight hours after transfection, cell lysates were assayed for firefly luciferase (from pG5-luc) and Renillar luciferase (from pBIND) activities using the Dual-Luciferase Reporter Assay System (Promega). Values were normalized to that of the empty vector control. All transfection experiments were performed in triplicate and repeated at least 3 times with similar results.

Coimmunoprecipitation and immunoblot analysis. To make the GST fusion protein, mouse Spdef cDNA was amplified using primers (forward, 5' GGGCACGGATCCATGGGCAGTGCCAGCCCAGG-3'; reverse, 5'CCCGGGGTCGACTCAGACTGGATGCACAAATTGGTAG-3'). The PCR product was digested with BamHI and SalI (shown in bold), subcloned into the pGEX4T-1 GST vector (Amersham Biosciences), and transformed into B21 bacteria for protein expression. After 5 hours of incubation at $37^{\circ} \mathrm{C}$ in $1 \mathrm{mM}$ isopropyl- $\beta$-D-thiogalactoside, bacteria were harvested and stored at $-80^{\circ} \mathrm{C}$ overnight. Cells were resuspended in $1 \times$ PBS, sonicated, treated with $1 \%$ Triton X-100 for 30 minutes on ice, and centrifuged at $12,000 \mathrm{~g}$ for 10 minutes. The protein was purified on glutathione-Sepharose. Eluted SPDEF was then dialyzed against $10 \mathrm{mM}$ Tris- $\mathrm{HCl}(\mathrm{pH} 8.0)$ at $4{ }^{\circ} \mathrm{C}$ and stored at $-80^{\circ} \mathrm{C}$. 3XFLAG-TTF- 1 and 3XFLAG 3 (aa 159-372) were described previously (19). 3XFLAG $\Delta 14$ (aa 1-221) was generated by PCR amplification of the coding region of $\Delta 14$ TTF- 1 and subcloned into the HindIII/BamHI sites of the 3XFLAG CMV-10 vector (Sigma-Aldrich). HeLa cells were plated at a density of $1 \times 10^{5}$ in 6-well plates and transfected with 3 XFLAG-TTF-1, 3XFLAG $\Delta 14$, and $3 X F L A G \Delta 3$. Forty-eight hours after transfection with Effectene, nuclear extracts were prepared as previously described (19). Coimmunoprecipitation was performed by incubating GST or GST-SPDEF proteins bound to glutathione-agarose beads with nuclear extract prepared from HeLa cells transfected with FLAG-TTF-1 constructs (19). Proteins were eluted by resuspending the beads directly in SDS-PAGE sample buffer and heating at $100^{\circ} \mathrm{C}$ for 5 minutes before loading on gels. Proteins from lysates of HeLa cells transfected with an SPDEF expression plasmid, as well as protein from mouse tracheae and lungs, were prepared as described above 
and separated by SDS-PAGE. Proteins were transferred to nitrocellulose and detected with guinea pig polyclonal antibody generated against recombinant mouse SPDEF.

Dust mite allergen exposure in IL-13 and Stat- $6^{-1-}$ transgenic models. Animals were maintained and handled under procedures approved by the Institutional Animal Care and Use Committee of the Cincinnati Children's Hospital Medical Center and in accordance with the Guide for the Care and Use of Laboratory Animals (National Research Council). Control C57BL/ 6 and Stat- $6^{-/-}$mice were treated intratracheally with IL-13 as previously described (9). Lung tissue was kindly provided by R. Finkelman (University of Cincinnati). IL-13 was expressed under conditional control in CCSP-rtTA/tetO-CMV-IL-13 transgenic mice as previously reported (9). Lung tissue was kindly provided by M. Rothenberg and P. Fulkerson (Cincinnati Children's Hospital Medical Center). The expression of the IL-13 transgene was induced by treatment of the mice with doxycycline. On days 0 and 7, 4-week-old $I L$-13-deficient mice on BALB/c background (kindly provided by A. McKenzie, Medical Research Council Laboratory of Molecular Biology, Cambridge, United Kingdom; ref. 46) and 3- to 5-week-old wild-type BALB/c mice (The Jackson Laboratory) were sensitized intraperitoneally with $10 \mu \mathrm{g}$ of house dust mite (HDM; Greer Laboratories) in $100 \mu \mathrm{l}$ PBS or equivalent amount of PBS alone. On days 14 and 21, mice were anesthetized intraperitoneally with a mixture of ketamine and xylazine (Phoenix Pharmaceutics Inc.) and challenged intratracheally with $100 \mu \mathrm{g}$ HDM in $50 \mu \mathrm{l}$ PBS or PBS alone. On day
26, the lung tissue was harvested and fixed with $10 \%$ neutral formalin (Sigma-Aldrich). The lung tissue was embedded in paraffin, and $5-\mu \mathrm{m}$ sections were cut for histological analysis.

Statistics. Data from morphometric analyses of airway segments were analyzed by a 1-tailed Student's $t$ test of the means for pairwise comparisons. Mann-Whitney rank sum test was used if data did not pass initial test of normality. Overall comparison group differences were assessed by Kruskal-Wallis 1-way ANOVA on the ranks. Luciferase data were compared using ANOVA.

\section{Acknowledgments}

This work was funded by grants HL56387 and HL38859, and the authors would like to thank Paula Blair, David Loudy, Gail Macke, Weiguo Chen, and William Hull for technical help and Ann Maher for secretarial assistance.

Received for publication May 22, 2006, and accepted in revised form January 10, 2007.

Address correspondence to: Jeffrey A. Whitsett, Cincinnati Children's Hospital Medical Center, Section of Neonatology, Pulmonary and Perinatal Biology, 3333 Burnet Avenue, Cincinnati, Ohio 45229-3039, USA. Phone: (513) 636-4830; Fax: (513) 636-7868; E-mail: jeff.whitsett@cchmc.org.
1. Jackson,A.D. 2001. Airway goblet-cell mucus secretion. Trends Pharmacol. Sci. 22:39-45.

2. Nadel, J.A., and Burgel, P.R. 2001. The role of epidermal growth factor in mucus production. Curr. Opin. Pharmacol. 1:254-258.

3. Rogers, D.F. 2004. Airway mucus hypersecretion in asthma: an undervalued pathology? Curr. Opin. Pharmacol. 4:241-250.

4. Bachurski, C.J., Yang, G.H., Currier, T.A., Gronostajski, R.M., and Hong, D. 2003. Nuclear factor I/ thyroid transcription factor 1 interactions modulate surfactant protein C transcription. Mol. Cell. Biol. 23:9014-9024.

5. Chen, J., Knowles, H.J., Hebert, J.L., and Hackett, B.P. 1998. Mutation of the mouse hepatocyte nuclear factor/forkhead homologue 4 gene results in an absence of cilia and random left-right asymmetry. J. Clin. Invest. 102:1077-1082.

6. Kimura, S., et al. 1996. The T/ebp null mouse: thyroid-specific enhancer-binding protein is essential for the organogenesis of the thyroid, lung, ventral forebrain, and pituitary. Genes Dev. 10:60-69.

7. Liu, C., Morrisey, E.E., and Whitsett, J.A. 2002. GATA-6 is required for maturation of the lung in late gestation. Am. J. Physiol. Lung Cell. Mol. Physiol. 283:L468-L475.

8. Mucenski, M.L., et al. 2005. $\beta$-Catenin regulates differentiation of respiratory epithelial cells in vivo. Am. J. Physiol. Lung Cell. Mol. Physiol. 289:L971-L979.

9. Wan, H., et al. 2004. Foxa2 regulates alveolarization and goblet cell hyperplasia. Development. 131:953-964.

10. Wan, H., et al. 2004. Foxa2 is required for transition to air breathing at birth. Proc. Natl. Acad. Sci.U. S. A. 101:14449-14454.

11. Wan, H., et al. 2005. Compensatory roles of Foxa 1 and Foxa2 during lung morphogenesis. J. Biol. Chem. 280:13809-13816.

12. Yang, H., Lu, M.M., Zhang, L., Whitsett, J.A., and Morrisey, E.E. 2002. GATA6 regulates differentiation of distal lung epithelium. Development. 129:2233-2246.

13. Bohinski, R.J., DiLauro, R., and Whitsett, J.A. 1994. The lung-specific surfactant protein B gene promoter is a target for thyroid transcription factor 1 and hepatocyte nuclear factor 3 , indi- cating common factors for organ-specific gene expression along the foregut axis. Mol. Cell. Biol. 14:5671-5681.

14. Bruno, M.D., Bohinski, R.J., Huelsman, K.M., Whitsett, J.A., and Korfhagen, T.R. 1995. Lung cell-specific expression of the murine surfactant protein $\mathrm{A}(\mathrm{SP}-\mathrm{A})$ gene is mediated by interactions between the SP-A promoter and thyroid transcription factor-1 [erratum 1995, 270:16482]. J. Biol. Chem. 270:6531-6536.

15. Kelly, S.E., Bachurski, C.J., Burhans, M.S., and Glasser, S.W. 1996. Transcription of the lungspecific surfactant protein $C$ gene is mediated by thyroid transcription factor $1 . \mathrm{J}$. Biol. Chem. 271:6881-6888.

16. Ray, M.K., Chen, C.Y., Schwartz, R.J., and DeMayo, F.J. 1996. Transcriptional regulation of a mouse Clara cell-specific protein (mCC10) gene by the NKx transcription factor family members thyroid transcription factor 1 and cardiac muscle-specific homeobox protein (CSX). Mol. Cell. Biol. 16:2056-2064.

17. Liu, C., Glasser, S.W., Wan, H., and Whitsett, J.A. 2002. GATA- 6 and thyroid transcription factor- 1 directly interact and regulate surfactant protein-C gene expression. J. Biol. Chem. 277:4519-4525.

18. Naltner, A., Ghaffari, M., Whitsett, J.A., and Yan, C. 2000. Retinoic acid stimulation of the human surfactant protein B promoter is thyroid transcription factor 1 site-dependent. J. Biol. Chem. 275:56-62.

19. Park, K.S., et al. 2004. TAZ interacts with TTF-1 and regulates expression of surfactant protein-C. J. Biol. Chem. 279:17384-17390.

20. Yan, C., Naltner, A., Conkright, J., and Ghaffari, M. 2001. Protein-protein interaction of retinoic acid receptor alpha and thyroid transcription factor- 1 in respiratory epithelial cells. J. Biol. Chem. 276:21686-21691.

21. Yi, M., Tong, G.X., Murry, B., and Mendelson, C.R. 2002. Role of CBP/p300 and SRC- 1 in transcriptional regulation of the pulmonary surfactant protein-A (SP-A) gene by thyroid transcription factor-1 (TTF-1). J. Biol. Chem. 277:2997-3005.

22. Brody, S.L., Yan, X.H., Wuerffel, M.K., Song, S.K., and Shapiro, S.D. 2000. Ciliogenesis and left-right axis defects in forkhead factor HFH-4-null mice. Am. J. Respir. Cell Mol. Biol. 23:45-51.
23. deFelice, M., et al. 2003. TTF-1 phosphorylation is required for peripheral lung morphogenesis, perinatal survival, and tissue-specific gene expression. J. Biol. Chem. 278:35574-35583.

24. Oettgen, P., et al. 2000. PDEF, a novel prostate epithelium-specific ets transcription factor, interacts with the androgen receptor and activates prostate-specific antigen gene expression. J. Biol. Chem. 275:1216-1225.

25. Yamada, N., Tamai, Y., Miyamoto, H., and Nozaki, M. 2000. Cloning and expression of the mouse Pse gene encoding a novel Ets family member. Gene. 241:267-274.

26. Khoor, A., Gray, M.E., Hull, W.M., Whitsett, J.A., and Stahlman, M.T. 1993. Developmental expression of SP-A and SP-A mRNA in the proximal and distal respiratory epithelium in the human fetus and newborn. J. Histochem. Cytochem. 41:1311-1319.

27. Karim, F.D., et al. 1990. The ETS-domain: a new DNA-binding motif that recognizes a purine-rich core DNA sequence. Genes Dev. 4:1451-1453.

28. Liu, Y., Jiang, H., Crawford, H.C., and Hogan, B.L. 2003. Role for ETS domain transcription factors Pea3/Erm in mouse lung development. Dev. Biol. 261:10-24.

29. Lin, S., Perl, A.K., and Shannon, J.M. 2006. Erm/ thyroid transcription factor 1 interactions modulate surfactant protein C transcription. J. Biol. Chem. 281:16716-16726.

30. Perl, A.K., Tichelaar, J.W., and Whitsett, J.A. 2002. Conditional gene expression in the respiratory epithelium of the mouse. Transgenic Res. 11:21-29.

31. Perl, A.K., et al. 2005. Conditional recombination reveals distinct subsets of epithelial cells in trachea, bronchi, and alveoli. Am. J. Respir. Cell Mol. Biol. 33:455-462.

32. Jain-Vora, S., Wert, S.E., Temann, U.A., Rankin, J.A., and Whitsett, J.A. 1997. Interleukin-4 alters epithelial cell differentiation and surfactant homeostasis in the postnatal mouse lung. Am. J. Respir. Cell Mol. Biol. 17:541-551.

33. Kuperman, D.A., et al. 2002. Direct effects of interleukin-13 on epithelial cells cause airway hyperreactivity and mucus overproduction in asthma. Nat. Med. 8:885-889.

34. Rankin, J.A., et al. 1996. Phenotypic and physiolog- 
ic characterization of transgenic mice expressing interleukin 4 in the lung: lymphocytic and eosinophilic inflammation without airway hyperreactivity. Proc. Natl. Acad. Sci. U. S. A. 93:7821-7825.

35. Wills-Karp, M., et al. 1998. Interleukin-13: central mediator of allergic asthma. Science. 282:2258-2261.

36. Tomkinson, A., et al. 2001. Temporal association between airway hyperresponsiveness and airway eosinophilia in ovalbumin-sensitized mice. Am. J. Respir. Crit. Care Med. 163:721-730.

37. Chen, H., Nandi, A.K., Li, X., and Bieberich, C.J. 2002. NKX-3.1 interacts with prostate-derived Ets factor and regulates the activity of the PSA promoter. Cancer Res. 62:338-340.

38. Shroyer, N.F., Wallis, D., Venken, K.J., Bellen, H.J., and Zoghbi, H.Y. 2005. Gfi1 functions downstream of Math1 to control intestinal secretory cell subtype allocation and differentiation. Genes Dev.
19:2412-2417.

39. Guazzi, S., et al. 1990. Thyroid nuclear factor 1 (TTF-1) contains a homeodomain and displays a novel DNA binding specificity. EMBO J. 9:3631-3639.

40. Besnard, V., Wert, S.E., Kaestner, K.H., and Whitsett, J.A. 2005. Stage-specific regulation of respiratory epithelial cell differentiation by Foxa1. Am. J. Physiol. Lung Cell. Mol. Physiol. 289:L750-L759.

41. Li, D., Gallup, M., Fan, N., Szymkowski, D.E., and Basbaum, C.B. 1998. Cloning of the aminoterminal and 5 '-flanking region of the human MUC5AC mucin gene and transcriptional upregulation by bacterial exoproducts. J. Biol. Chem. 273:6812-6820.

42. Park, K.-S., Wells, J.M., Zorn, A.M., Wert, S.E., and Whitsett, J.A. 2006. Sox17 influences the differentiation of respiratory epithelial cells. Dev. Biol. 294:192-202.
43. Wert, S.E., Dey, C.R., Blair, P.A., Kimura, S., and Whitsett, J.A. 2002. Increased expression of thyroid transcription factor-1 (TTF-1) in respiratory epithelial cells inhibits alveolarization and causes pulmonary inflammation. Dev. Biol. 242:75-87.

44. Zhou, L., Lim, L., Costa, R.H., and Whitsett, J.A. 1996. Thyroid transcription factor-1, hepatocyte nuclear factor-3beta, surfactant protein B, C, and Clara cell secretory protein in developing mouse lung. J. Histochem. Cytochem. 44:1183-1193.

45. Wert, S.E., Glasser, S.W., Korfhagen, T.R., and Whitsett, J.A. 1993. Transcriptional elements from the human SP-C gene direct expression in the primordial respiratory epithelium of transgenic mice. Dev. Biol. 156:426-443.

46. McKenzie, G.J., et al. 1999. Impaired development of Th2 cells in IL-13-deficient mice. Immunity. 9:423-432. 